\title{
Hydrates of cyclobutylamine - modifications of gas clathrate types sI and sH
}

\author{
Lukasz Dobrzycki*, Kamila Pruszkowska, Roland Boese, Michat K. Cyrański \\ The Czochralski Laboratory of Advanced Crystal Engineering, Faculty of Chemistry, \\ University of Warsaw, Zwirki i Wigury 101, 02-089 Warsaw, Poland

\section{Supporting Information}

\section{Single crystal X-ray diffraction data processing}

Several experiments by in situ crystallization resulted in 'oligocrystalline' samples (many simultaneously diffracting crystal specimen) e.g. for neat amine, monohydrate and the $91 / 2$ hydrate. The data processing for the neat amine at both temperatures listed in the Table 1 was performed for the major domain with the HKLF5 option. For the monohydrate at $100 \mathrm{~K}$ two twin domains were applied generating a HKLF5 file, whereas the data at $202 \mathrm{~K}$ data were integrated with fixed and an integration box small enough so no twinning was needed to be taken into account. Thus, the data completeness at $100 \mathrm{~K}$ is slightly higher than at $202 \mathrm{~K}$ (94.5\% vs 92.0\%). During the structure refinement based on HKLF5 two twin component fractions were included. When the refinement had converged, reflections with identical/ 
equivalent indices were merged ${ }^{42}$ and the final refinement was performed with HKLF4 settings. The data integration and scaling for the $9 \frac{1}{2}$ hydrate was based on two components and generating a HKLF4 file, thus no further merging was necessary.

\section{Single crystal X-ray diffraction structure refinement}

Thermal ellipsoid plots at the 50\% probability level are displayed in Figure S1, Figure S2 and Figure S3.

Neat amine. In the case of the cBA at both temperatures (Table 1) all non-hydrogen atoms were refined anisotropically. Positions of hydrogen atoms of the amino groups were fully refined together with their isotropic displacement parameters. The other $\mathrm{H}$ atoms were placed in calculated positions and refined within the riding model. Their isotropic displacement parameters were set to the 1.2 fold of the $\mathrm{U}_{\text {eq }}$ applied to the corresponding heavy atom.

Monohydrate. For both temperatures (Table 1) the positions of the all non-hydrogen atoms were refined together with anisotropic displacement parameters. Positions of all hydrogen atoms engaged in $\mathrm{HBs}$ were refined, some with either $\mathrm{O}-\mathrm{H}$ or $\mathrm{N}-\mathrm{H}$ distance restraints. Isotropic displacement parameters of these atoms were either refined or set to the 1.5 fold of the $\mathrm{U}_{\mathrm{eq}}$ for the corresponding heavy atom for the measurements performed at $202 \mathrm{~K}$ and $100 \mathrm{~K}$, respectively. All aliphatic $\mathrm{H}$ atoms were placed in calculated positions and refined within the riding model. Their isotropic temperature parameters were set to the 1.2 fold of the $\mathrm{U}_{\text {eq }}$ for the corresponding heavy atom.

6 3/7 hydrate. The structure can be refined in two possible space groups: $P-3 m 1$ and $P 6 / \mathrm{mmm}$. The trigonal SG choice results in significantly lower discrepancy factors at both temperatures using a higher ratio diffraction data $v s$ refined parameters (see Table 1).

In the trigonal SG the structure contains three amine moieties located at special positions in the asymmetric part of the unit cell. All non-H atoms of these cBA molecules were refined anisotropically with some distance constraints modeling a reasonable geometry of the amine. 
The hydrogen atoms were assigned to most of the heavy atoms except for two amino groups. The displacement parameters of all $\mathrm{H}$ atoms were set either to the 1.2 fold or 1.5 fold of the $\mathrm{U}_{\mathrm{eq}}$ for the corresponding heavy atom. In the asymmetric part of the unit cell there are eight positions of water molecules with only one $\mathrm{H}_{2} \mathrm{O}$ molecule located in a general position. One of the water molecules is partially substituted by the amino group of a cBA molecule. This results in a smaller occupancy of this $\mathrm{H}_{2} \mathrm{O}$ molecule (sum occupancy equal 5/6 instead of 1 ). All the other $\mathrm{H}$ atoms for this partially occupied $\mathrm{H}_{2} \mathrm{O}$ molecule were not assigned and further $\mathrm{H}$ atoms were located as centroids between neighboring $\mathrm{O}$ atoms, placed at $0.95 \AA \mathrm{O}-\mathrm{H}$ distances which were not refined. All $\mathrm{H}_{2} \mathrm{O}$ molecules exhibit positional disorder of the $\mathrm{H}$ atoms as observed in general for natural gas clathrate hydrates. The structure refinements at both temperatures were based on the same model.

In contrast to the trigonal SG choice the number of refined parameter in the hexagonal $\mathrm{SG}$ is smaller which is associated with the higher symmetry of the atomic positions in the unit cell. However, the final structural model including the number of atoms is similar for both SG choices.

"7 $1 \frac{1}{2}$ " hydrate. The structure contains a non-stoichiometric amount of water molecules due to partial substitution of one of the $\mathrm{H}_{2} \mathrm{O}$ molecules by the amino group of cBA. Moreover, this non-fully occupied $\mathrm{H}_{2} \mathrm{O}$ molecule is disordered over two positions with occupancies equal to 0.800 and $0.076 \%$ (instead of 0.200 ). Applying a $100 \%$ sum occupancy of this molecule the final discrepancy factor increases significantly. Another water molecule is also disordered over two positions with an occupancy ratio equal to $0.955: 0.045$. The amine is even more disordered with three alternative positions of $\mathrm{N}$ atoms $(0.488: 0.264: 0.248)$ located around 3fold axis of symmetry; including the disorder result in a reasonable displacement parameters for almost all the respective atoms, except of those with low occupancies. All the hydrogen 
atoms for the water molecules were treated as for the $63 / 7$ hydrate and the structure refinements were based on the same model for both temperatures.

$9 \frac{1}{2}$ hydrate. Similarly to the $63 / 7$ hydrate this structure can be refined either in the trigonal $P-31 \mathrm{~m}$ or hexagonal $P 6 / \mathrm{mmm}$ SG. In this case, however, the final discrepancy factors for the stronger diffracting data are slightly smaller for the trigonal refinement at lower temperatures whereas at $270 \mathrm{~K}$ the situation is reversed. The discrepancy factors for all reflections are slightly lower for the hexagonal SG choice and in the trigonal refinements the number of diffraction data per refined parameters is higher $-c a$. $11 v s c a$. 9. Similar to the $63 / 7$ hydrate the $9 \frac{1}{2} \mathrm{cBA}$ hydrate contains one of the water molecule which is partially substituted by the amine molecules. The occupancy of this atom was set to $1 / 3$ instead of $1 / 2$ (in triclinic SG) resulting in a decrease by two for the total number of water molecules in the unit cell. For all $\mathrm{O}$ atoms the coordinates and anisotropic displacement parameters were refined. The crystal lattice contains two amine molecules both located at special positions. All heavy atoms of one of the cBA molecules were refined with the anisotropic approach. No hydrogen atoms were located at amino groups, however for these two molecules three aliphatic $\mathrm{H}$ atoms were located. The positions of these atoms result from the superpositioning after transformation by the symmetry operations of the crystallographically independent parts of the cBA molecules. The positions and isotropic displacement parameters for these three $\mathrm{H}$ atoms were refined. For modeling the geometry of the amine molecules a few N-C and C-C distance constrains (additional C-H constrains were used for refinement of the structure at 270K) were applied. The hydrogen atoms of the water molecules were treated in the same manner as in the $63 / 7$ hydrate. In contrast to the trigonal SG choice, the number of refined parameter is smaller in the hexagonal SG what is due to the higher symmetry of the atomic positions in the unit cell. However, the final model including the sum formula is comparable for both choices of SGs. 
a) $\mathrm{cBA}$ at $100 \mathrm{~K}$

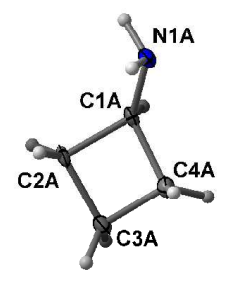

b) cBA at $170 \mathrm{~K}$
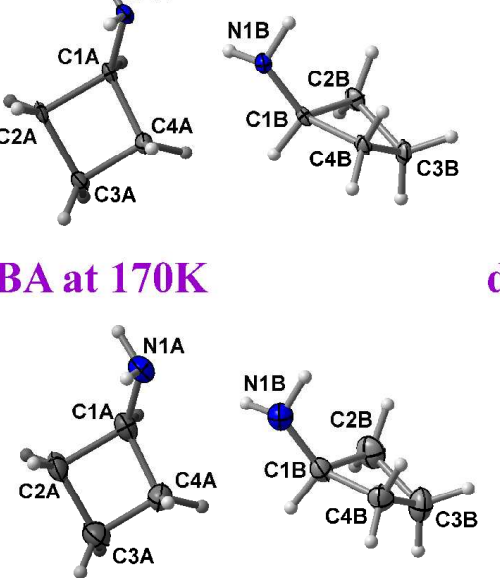

e) $63 / 7$ hydrate at $100 \mathrm{~K}$
in $\overline{P 3} m 1$

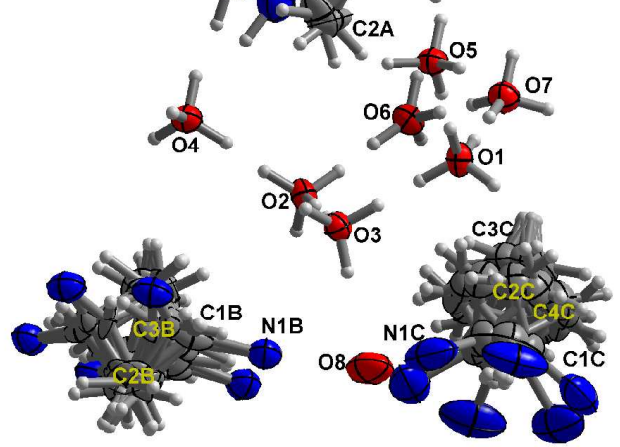

f) $63 / 7$ hydrate

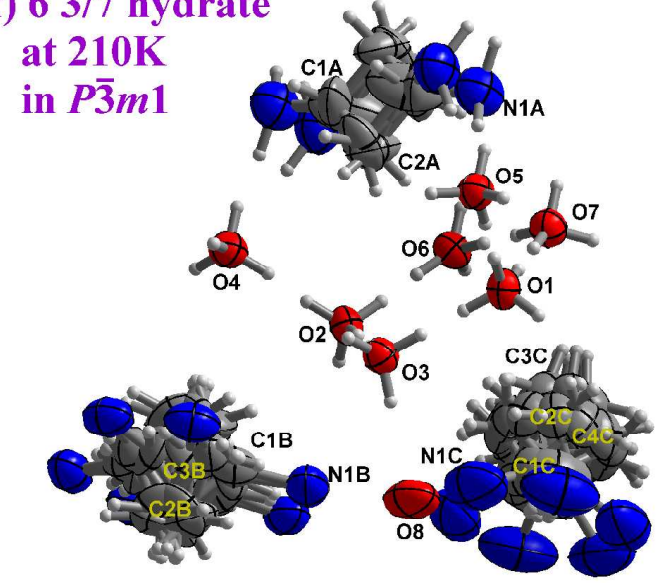

c) Monohydrate at $100 \mathrm{~K} 010 \mathrm{~g}-\frac{01 \mathrm{~B}}{8}$

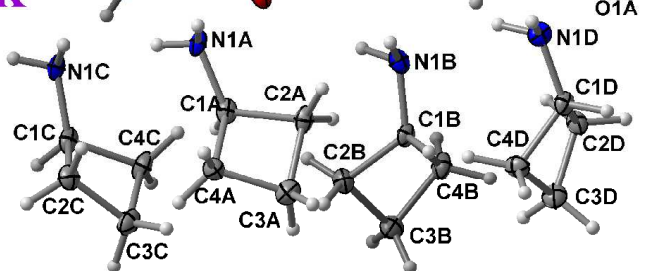

d) Monohydrate

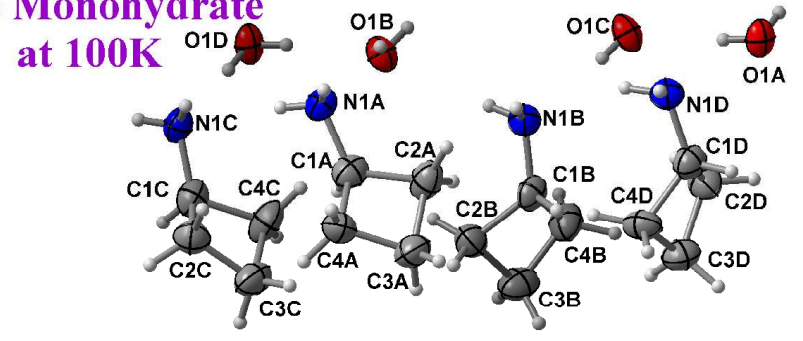

g) $63 / 7$ hydrate at $100 \mathrm{~K}$ in P6/mmm
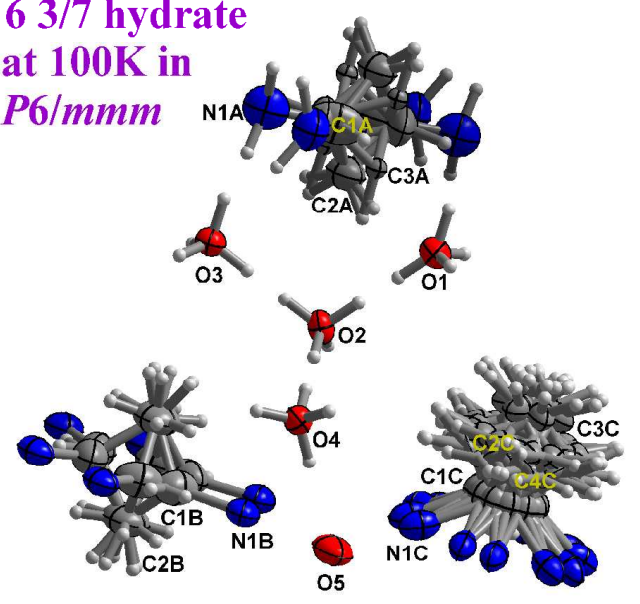

h) $63 / 7$ hydrate

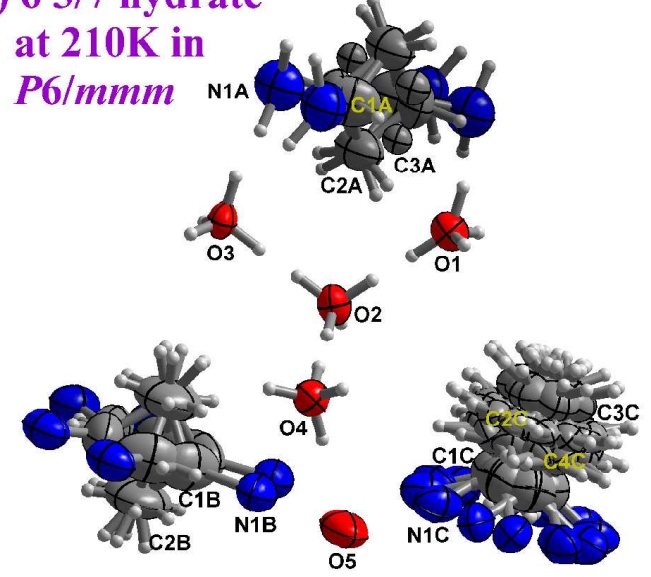

Fig. S1. Thermal ellipsoid plot at the $50 \%$ probability level. 
a) "7 1/2" hydrate at $100 \mathrm{~K}$

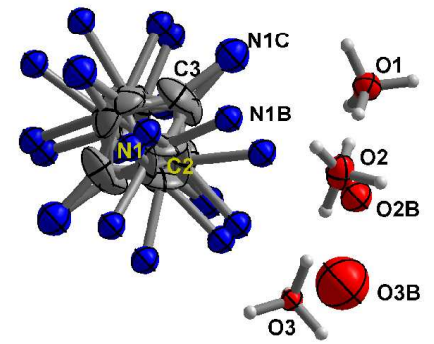

b) "7 1/2" hydrate at $195 \mathrm{~K}$

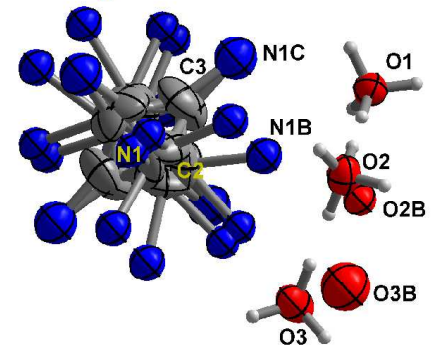

Fig. S2. Thermal ellipsoid plot at the $50 \%$ probability level. 
a) $91 / 2$ hydrate at $100 \mathrm{~K}$ in $P \overline{3} m 1$

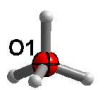

$03 \mathrm{y}=$

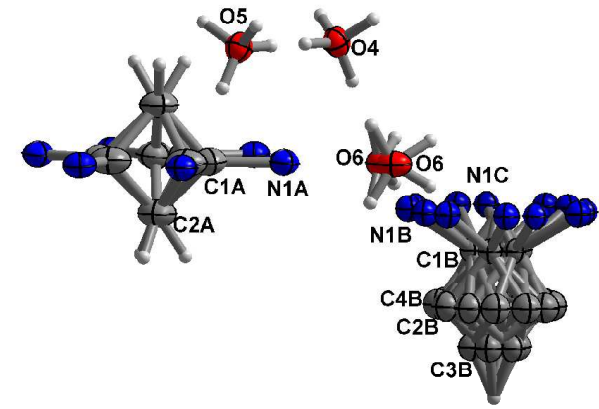

b) $91 / 2$ hydrate at $150 \mathrm{~K}$ in $P \overline{3} m 1$

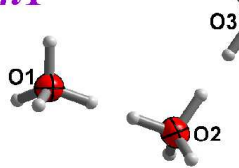
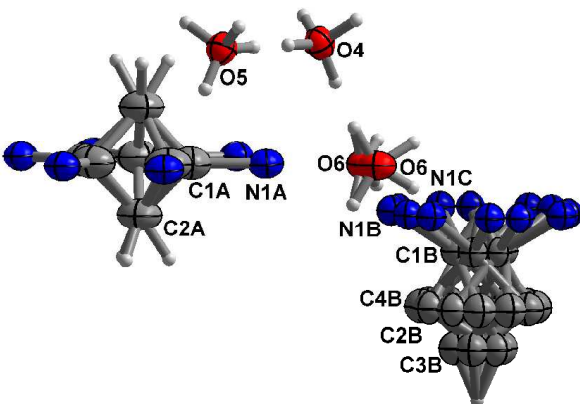

c) $91 / 2$ hydrate at $270 \mathrm{~K}$

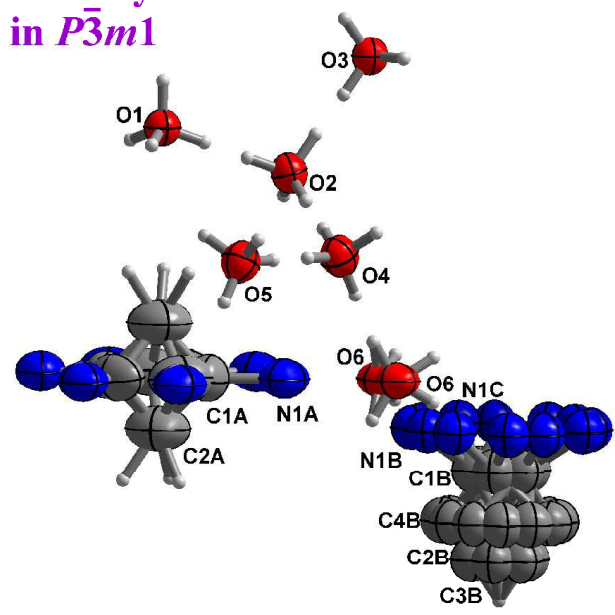

d) $91 / 2$ hydrate at $100 \mathrm{~K}$ in $P 6 / \mathrm{mmm}$

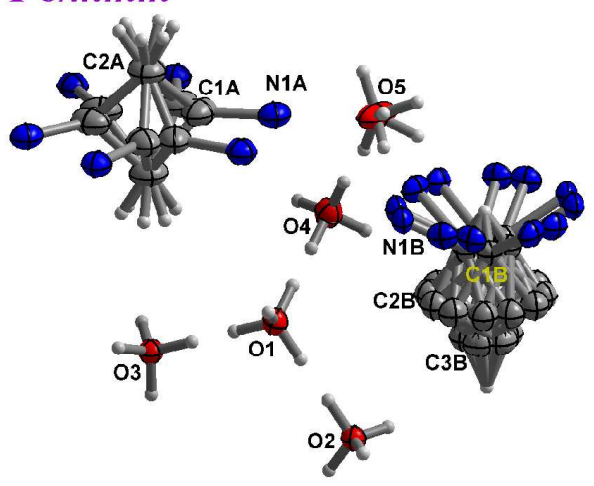

e) $91 / 2$ hydrate at $150 \mathrm{~K}$ in $P 6 / \mathrm{mmm}$

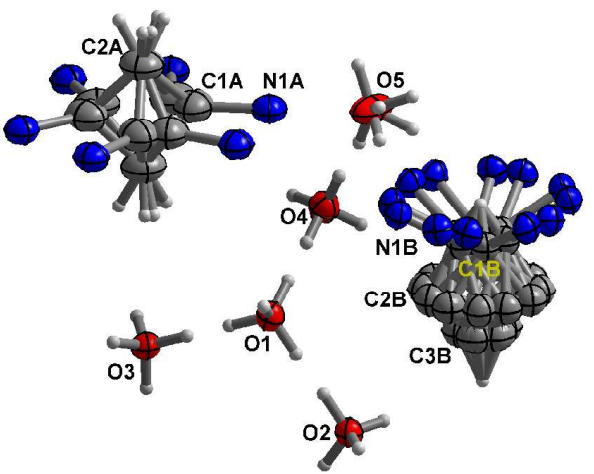

f) $91 / 2$ hydrate at $270 \mathrm{~K}$ in $P 6 / \mathrm{mmm}$

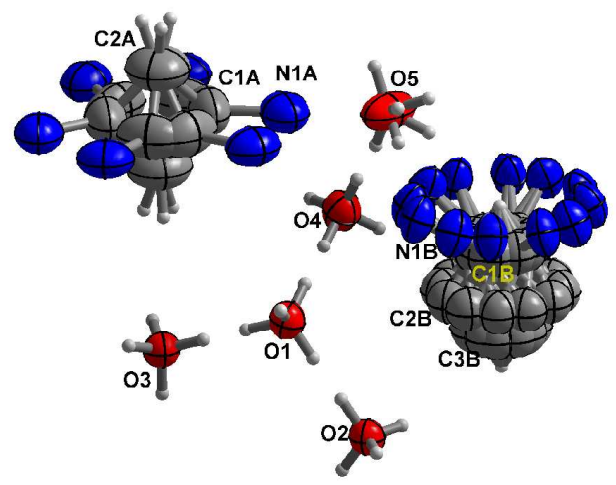

Fig. S3. Thermal ellipsoid plot at the $50 \%$ probability level. 


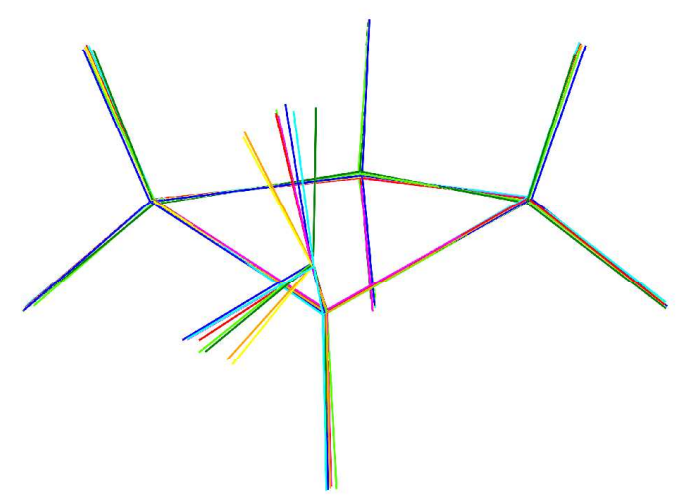

cBA, molecule B

hemihydrate, molecule 2

monohydrate, molecule A

monohydrate, molecule C

monohydrate, molecule D

Fig. S4. Overlay of the amine molecules from the structures of the neat compound, hemihydrate (ref. 13) and monohydrate. 